\title{
The Potential for the Application of X-ray Photoelectron Spectroscopy in Forensic Science
}

\author{
John F Watts \\ Surrey Materials Institute and \\ Faculty of Engineering \& Physical Sciences \\ UNIVERSITY OF SURREY \\ Guildford Surrey GU2 7XH UK
}

\author{
j.watts@surrey.ac.uk
}

\begin{abstract}
The potential for the use of X-ray photoelectron spectroscopy (XPS) in forensic science is reviewed, taking as a starting point a series of examples and case histories this review speculates on other areas where XPS may be able to make a contribution. The topics that are considered include chemical speciation of fingerprints, thin layers deposited on substrates as a result of explosions and/or fire, particulate materials and cosmetics and finally sequestration of marker elements and molecules on surfaces. It is concluded that XPS has much to offer the forensic science community but investigations may be hampered by the need to compare crimes scene specimens with known standards. Until this issue is addressed it is likely that XPS will continue to be used on an ad hoc basis for particularly challenging specimens in high profile cases.
\end{abstract}




\section{Introduction}

It is now some forty years since XPS became available as a commercial technique and over this time the technique has developed into a mature analytical technique widely encountered in both corporate and academic research laboratories. In the intervening years XPS has made important contributions to the advancement of knowledge in many areas such as physics, chemistry, materials science, electronics to name but a few. As the technique became more widely known (the mid to late 1970s) so its role began to develop from an analytical method used in well defined research programmes to one that was used to gather information that could indicate the cause of failure of a product or structure. In essence XPS was being used as a forensic technique to analyse surfaces associated with failure or poor performance of the material in question.

By the mid 1980s XPS could be said to have been used in a forensic capacity in a number of areas: adhesive bonding, organic coatings, corrosion, catalysis, polymer surface treatment, metallurgy and so forth. Given this distinguished history of application in the forensic analysis of failure surfaces it is somewhat surprising that its use in forensic science, associated with the examination and speciation of specimens gathered at the scene of a crime, is very low indeed. The reasons for this are manifold but the main reasons must be; the need for the analysis of samples within a very short timescale, without standard samples for comparison; the cost of such an analysis; and the initial large area nature of the analysis (many square millimetres). Although XPS is traditionally regarded as a large area analysis method, the last 25 years have seen small area XPS analysis improve by more than an order of magnitude from $250 \mu \mathrm{m}$ to $15 \mu \mathrm{m}$. Indeed many analysts now consider a large area analysis to be an area of $500 \mu \mathrm{m}$ diameter.

The aim of this brief paper is to review the attributes of XPS alongside the requirements of an analytical technique that will be of value to the forensic scientist. This will be followed by areas in which XPS has already made a contribution to forensics, and the potential for expanding such application areas.

\section{XPS as an Analytical Technique for Forensic Science}

The advantages to be accrued from XPS analysis will be well known to readers of this journal but bear repeating to help assess the technique in context of forensic science. XPS has a characteristic analysis depth of ca. $5 \mathrm{~nm}$ and is able to detect extremely small quantities of material on a solid surface $(<10 \%$ of a monolayer). The determination of a quantitative surface chemical analysis is straightforward as is the provision of chemical state information for all elements in the periodic table from helium onwards. Angle resolved XPS (ARXPS) can be employed to probe different analysis depths and can be used to provide a quantitative compositional depth profile of the outer $5 \mathrm{~nm}$ or so. The thickness of overlayers within the XPS analysis depth is readily determined and the hierarchy of a series of layers can be deduced by ARXPS or the consideration of the background of inelastically scattered electrons. XPS can be applied to all (vacuum compatible) materials, tobacco, wood and food products are some of the more unusual materials analysed in the author's laboratory in recent years. XPS images are also possible using modern systems although the resolution is significantly less than that 
achieved by scanning electron microscopy combined with energy dispersive X-ray analysis (SEM/EDX), Auger electron spectroscopy (AES) or time of flight secondary ion mass spectrometry (ToF-SIMS), but the strengths of XPS enable maps of derived properties (such as the thickness of a surface phase) to be presented ${ }^{1}$. In terms of sample preparation as long as the specimen can be accommodated in the analysis chamber of the spectrometer it can be retained, undamaged and unaltered, as evidence and for re-analysis if necessary. Samples that are more than a few centimetres square will probably need to be sectioned but some modern spectrometers can handle very large specimens indeed.

Such advantages must be set against the potential disadvantages of the technique, its limited spatial resolution, the need for exposure of the sample to an ultra high vacuum and the high capital cost of the instrument, although several manufacturers are now marketing reasonably priced, but high performance, entry level systems. Such systems tend to restrict the flexibility of the hardware although not at the cost of performance.

So how effectively do these characteristics map onto the requirements of the forensic scientist? In the category of thin layers on solid surfaces there are two potential applications that spring to mind; fingerprints and the chemical definition of thin layers resulting from fire or explosions. Elemental analysis may be sufficient to associate such a layer with a potential source whilst chemical state information may provide clues as to changes that have occurred during the fire or explosion, or identify personal care products applied by the suspect and left within the latent fingerprint. Particulate samples, providing there is a sufficient amount available need care in their mounting for analysis. The best methods for mounting such samples are dusting onto adhesive tape or embedding in indium foil, compacting is not recommended as entrapped air can sometimes lead to specimens disintegrating in the vacuum chamber and contamination of the sealing surfaces of valves and the like. Once presented successfully for analysis the prognosis for successful analysis is high with the level of information far superior to that of SEM/EDX. Such methods may be particularly useful in a supporting role in differentiating between ostensibly similar cosmetic products. In a similar vein the analysis of hair by XPS allows the identification of certain life-style and/or personal care choices to be made.

Surfaces of solids can sometimes act as effective sites for the sequestration of minute amounts of materials that may provide clues to the source of the material. XPS provides an easy way to identify and quantify such sequestrants both on materials where their deposition is by chance or on those specifically intended for the purpose such as biosensors. The strength of XPS in such applications is the ease of presentation of such samples for analysis with no need to extract the small amount of material from the sample surface.

It should be noted that in the 14th International Forensic Science Conference ${ }^{2}$ (the Proceedings of which run 585 pages) XPS was cited just three times in the context of glass, soil, and photocopier toner investigations. This indicates that the forensic science community regards XPS as a peripheral technique at best, probably for the reasons outlined above. The following sections will seek to redress the balance a little with the 
identification of potential areas of forensic science to which XPS is able to make a contribution. This will consider the past and the potential utility of XPS in forensic science.

\section{Fingerprint Identification}

Fingerprints have been analysed since the early days of XPS, not for forensic reasons, in fact just the opposite, to indicate to the layman the very surface specific nature of the analysis technique. This is a "party piece" in many surface analysis laboratories including that of the author. The usual procedure is to take a clean metal surface (aluminium foil straight from the roll is ideal) and mount this for analysis. A duplicate piece of foil is prepared and fingered lightly by the operative or the VIP visitor to the laboratory. XPS analysis then reveals the clean sample as having a modest $\mathrm{C} 1 \mathrm{~s}$ peak and characteristic metal peaks and O1s spectrum which are very intense. Analysis of the sample with the fingerprint will show a very intense $\mathrm{C} 1 \mathrm{~s}$ line, probably total attenuation of the metal peaks and small concentrations of sodium and chlorine. Such an experiment is very effective in indicating the importance of careful specimen preparation and handling prior to XPS analysis but it hardly makes a major contribution to forensic science!

However the first suggestion that XPS could be used for forensic purposes was the result of such an experiment, performed for the local press by David Clark and David Briggs in 1970 , and the total misunderstanding by the journalist of the scientific point that was being made. The report, carried in the UK's Northern Evening News in the autumn of 1970 is reproduced in Figure 1 and the title and accompanying copy make it clear that the journalist understood (incorrectly of course) how the new technique of XPS would stop burglars in their tracks! Buried at the end of the piece is a comment by David Clark to the effect that "Once the fundamental work has been done, I can see this machine being used routinely by industrialists all over the world" which it has indeed turned out to be the case.

Thus a latent fingerprint, when analysed by XPS, has apparently no specific or generic attributes in the spectrum that can aid with offender profiling, much less provide the absolute identification associated with the well established visual examination based on analysis, comparison, evaluation and verification used in the match of a fingerprint from a crime scene with one on file. There is one area in which XPS does have potential to assist in offender profiling, in that small residues of a variety of materials on the fingers will invariably be transferred to the print. This concept is illustrated by the following example in which the experiment of a fingerprint on aluminium foil gave unexpected results. The XPS survey spectra of Figure 2 show clean aluminium foil and foil that has been fingered in the manner described above. The clean aluminium is as expected but the aluminium with the fingerprint is unusual in that the carbon concentration is not high enough to attenuate the Al2p and Al2s peaks, and Si2p and Si2s peaks are seen in the spectrum. The solution is simple although not immediately obvious: The visitor to the laboratory on this occasion was a lady who was the local Member of Parliament, examination of the Si2p binding energy indicated that the silicon was present as an 
organic silicone, which suggest a silicone oil used in personal care products such as hand cream.

In this way XPS provides a potential route to identifying the use of certain products by the suspect, the quantitative nature of XPS providing an additional degree of certainty in the analysis. This concept can be extended further using ToF-SIMS, where both the latent image and the molecular nature of the residue can be determined ${ }^{3,4}$.

\section{Thin Overlayers on Solid Surfaces}

Certain activities, particularly those associated with terrorist bombs and arson attacks may give rise to the volatilisation or production of degradation of products which may then be deposited as a very thin film (nanometres in thickness) throughout the region effected by the fire or explosion. Identification of these thin residues may provide an important clue in the identification of the modus operandi of those responsible for the perpetration of the crime.

One example that falls into this category was the assassination of a senior UK politician in spring 1979. The bomb, attached to his car, was activated by a tiltswitch when the car started to move up an incline. The tiltswitch was a mercury based device and the liquid metal was volatilised in the subsequent explosion. XPS analysis of pieces of the damaged car established the presence of mercury on the surface and enabled the type of device to be identified. This played an important part in the subsequent investigation as the use of a mercury loaded tiltswitch was associated, at the time, with one particular terrorist group.

In a similar vein the identification of small amounts of degradation products may be detected and quantified on surfaces associated with an explosion or fire but in this case careful experiments will need to be undertaken to ensure that XPS standards of degradation products produced under controlled conditions, such as those described byVarga et $\mathrm{al}^{5}$, are available for comparison with data recorded from samples taken from the crime scene.

Another avenue worthy of investigation is the use of knowledge of the growth of oxide layers on metals as a function of temperature to be used as a means of estimation of the temperature that has been reached within various location in a fire scene. XPS is able to identify metal oxides very precisely and the appearance of one oxide rather than another, e.g copper(I) oxide is the lower temperature form of the oxide and copper (II) oxide forms at elevated temperatures, cupronickel coinage has a $\mathrm{NiO}$ terminal layer but on heating this is replaced by $\mathrm{CuO}$, other alloys such as stainless steel display similar characteristics. Although not explored as forensic methodologies all have potential for use in specific applications.

\section{Particulate Materials}

Particulate materials can be classified in a great many ways; shape, size, composition, but the most important attribute as far as surface analysis is concerned is the quantity that is available. A single particle will not be amenable to XPS analysis unless it is fairly large, 
at least many tens of micrometres, however a smear of particles comprising of mud, or cosmetics residue has a much greater chance of success, and XPS will come into its own with ultrafine particles in this situation as the material of the particulate itself can be analysed without interference from materials onto which the smear is deposited. For the analysis of single particulates ToF-SIMS or AES are more appropriate and in the early days of the detection of particulate debris on microelectronic devices AES was certainly the method of choice for the examination of very small particles. There are considerable issues in the analysis of insulating materials by AES but the current state of the art and ways in which such analyses can be achieved have recently been reviewed by Baer et el ${ }^{6}$

Recent work has shown that XPS may have an important role to play in the differentiation between cosmetic materials that are ostensibly comprised of the same material ${ }^{7}$. The following data ${ }^{8}$ presents the results of XPS analysis (Thermo Scientific Theta Probe) of a series of talcum powders (talc, a hydrated magnesium silicate, is a very soft mineral, the softest on the Moh's Scale of Hardness) from different manufacturers selected at random and a series of face powders. A representative XPS survey spectrum of talcum powder is shown in Figure 3 and the quantitative surface analyses of all the talcum powder samples are given in Table 1.

The mineralogical origins of talcum powder are clear from the quantitative surface analyses of Specimens 1 - 5 with all samples showing magnesium, silicon and oxygen from the mineral with a small amount of carbon contamination (the low level of hydrocarbon is, incidentally, very typical of a high surface area material such as a finely divided solid). As observed in Ref 5 the values of the $\mathrm{Mg} / \mathrm{Si}$ ratio changes with the source of the talcum powder. This may provide a powerful means of matching a sample of forensic significance against a particular brand,. In the calculation of $\mathrm{Mg} / \mathrm{Si}$ ratios there are three $\mathrm{Mg}$ core levels that are available to the analyst; $\mathrm{Mg} 1 \mathrm{~s}, \mathrm{Mg} 2 \mathrm{p}$ and $\mathrm{Mg} 2 \mathrm{~s}$. The usual practice in quantitative XPS is to use the $\mathrm{Mg} 1 \mathrm{~s}$ orbital which has a much larger cross section than the $\mathrm{Mg} 2 \mathrm{p}$ and $2 \mathrm{~s}$ lines. One slight drawback is that the electron attenuation length of the $\mathrm{Mg} 1 \mathrm{~s}$, which as a result of its lower kinetic energy, is $1.8 \mathrm{~nm}$ which is significantly lower that that of the Mg2p $(7.9 \mathrm{~nm})$ and $\mathrm{Mg} 2 \mathrm{~s}(8.2 \mathrm{~nm})$. The result of these effects is clearly seen in the $\mathrm{Mg} / \mathrm{Si}$ ratios of Table 1 which are calculated for all three $\mathrm{Mg}$ orbitals compared with the $\mathrm{Si} 2 \mathrm{p}$. As the $\mathrm{Mg} 2 \mathrm{~s}$ is closest in kinetic energy to the $\mathrm{Si} 2 \mathrm{p}$ the recommendation is to use this orbital as the analysis depths are very similar. One should be aware, however, that as the Mg2p and Si2p core levels have relatively low cross-sections in XPS, rather large errors might accrue on these quantitative calculations. If good quality high resolution spectra are used (as in the data presented in Table 1 \& 2) a value of $+/-5 \%$ in the quantitative analysis would be anticipated, but if data taken directly from the survey spectra is used a value as high as $+/-10 \%$ might result, depending on quality of spectra and so forth.

The face powder samples are clearly more complex formulations which may vary from manufacturer to manufacturer depending on the required properties, pigmentation and so forth. The potential for forensic analysis once again lies in the comparison of relevant samples against those with will well defined provenance. 
The advantage of XPS in these two example lies with the ease of sample preparation, the danger of damage to the sample on removal from the substrate is avoided as, providing the substrate can be sectioned for entry into the spectrometer, particles are not disturbed and the fact that the specimen is not destroyed or changed in any way ensures that it is available for further analysis if required. Subtle differences in the surface composition of similar sample are reflected in the XPS analysis but may not be apparent with a bulk analysis technique such as EDX.

The issue of identification of personal care products is also relevant in the analysis of samples of hair. Hair can carry trace element information that may provide valuable clues concerning the identity of the "donor". For instance heavy smokers are known to have trace quantities of arsenic in their hair which can be determined by XPS. Similarly the nature of personal care products used may be deduced from the XPS spectra, although the best results in this instance will be obtained by combining XPS data (to deduce coverage of such products on the surface) with ToF-SIMS to provide molecular identification.

\section{Sequestration of Marker Elements and Molecules on Surfaces}

The concept of using XPS to identify and quantify the amount of material immobilised on a solid surface is well established in certain fields and in recent years this approach has been used by those working in biosensors to validate the amount of the target material that has been immobilised 9 . As a surface will always, to a certain extent, reflect the environment to which it has been exposed there is the potential for using such evidence in a forensic manner. In the days when leaded automobile fuel was used in the UK leaves taken from motorway environments showed a clear indication of lead in the surface analysis by XPS whereas those from country environments did not.

\section{Conclusions}

All the indications are that at present XPS is little used in forensic science, as evidenced by the dearth of applications in the published literature, although the potential for it to contribute in certain areas is quite significant. This is in contrast to SEM/EDX where the method has become quite routine for forensic use ${ }^{10}$. It is likely that such promise will only be fulfilled once XPS has been successfully applied in cases where conventional chemical analysis methods have not been successful. In many cases there is a need to compare forensic specimens with samples of products in the marketplace. This is a very resource intensive undertaking and one which will require the enthusiastic support of funding agencies, the forensic science community and those in surface analysis. Until then one suspects that XPS will be used in forensic science on an ad hoc basis for particularly challenging analytical problems in high profile cases.

\section{Acknowledgements}

The author is grateful to Dr Marie-Laure Abel for bringing some of the literature to his attention, Dr Steve Hinder for recording XPS data of talcum and face powders especially for this review, and all in the Faculty of Engineering and Physical Sciences at the University of Surrey who provided samples of their cosmetics for analysis! Prof Dave Briggs was kind enough to supply the press cutting used as Figure 1. 
Table 1. Quantitative surface analysis by XPS of a series of talcum powders.

\begin{tabular}{|c|c|c|c|c|c|c|c|c|}
\hline \multirow{2}{*}{ Sample } & \multicolumn{4}{|c|}{ Surface Composition/atomic \% } & \multicolumn{3}{c|}{ Mg/Si Atom Ratios } \\
\cline { 2 - 9 } & $\mathrm{C}$ & $\mathrm{O}$ & $\mathrm{Si}$ & $\mathrm{Mg}$ & other & $\mathrm{Mg} 1 \mathrm{~s}$ & $\mathrm{Mg} 2 \mathrm{~s}$ & $\mathrm{Mg} 2 \mathrm{p}$ \\
\hline 1 & 6.1 & 53.6 & 11.7 & 28.6 & & 2.40 & 0.86 & 0.95 \\
\hline 2 & 8.2 & 52.6 & 10.7 & 28.5 & & 2.66 & 0.95 & 1.04 \\
\hline 3 & 6.2 & 52.6 & 10.7 & 28.5 & & 2.37 & 0.82 & 0.91 \\
\hline 4 & 10.2 & 53.5 & 12.0 & 28.5 & & 2.14 & 0.79 & 0.91 \\
\hline 5 & 9.2 & 52.8 & 10.9 & 26.6 & $\mathrm{~F}=0.4$ & 2.44 & 0.93 & 1.01 \\
\hline
\end{tabular}

Table 2. Quantitative surface analysis by XPS of body and face powders.

\begin{tabular}{|c|c|c|c|c|c|c|c|c|c|}
\hline \multirow{2}{*}{ Sample } & \multicolumn{10}{|c|}{ Surface Composition/atomic \% } \\
\cline { 2 - 11 } & $\mathrm{C}$ & $\mathrm{O}$ & $\mathrm{Si}$ & $\mathrm{Mg}$ & $\mathrm{Al}$ & $\mathrm{K}$ & $\mathrm{Na}$ & $\mathrm{N}$ & Others \\
\hline 1 & 18.3 & 58.1 & 9.1 & 0.5 & 9.3 & 3.0 & 0.7 & 0 & $\mathrm{Ca}=0.4$ \\
\hline 2 & 29.2 & 41.4 & 10.4 & 9.2 & 0 & 0 & 0 & 1.0 & $\mathrm{~F}=7.4$ \\
\hline 3 & 17.1 & 55.8 & 9.8 & 0 & 9.2 & 3.1 & 2.6 & 0.4 & $\mathrm{Fe}=2.1$ \\
\hline
\end{tabular}




\section{Figures:}

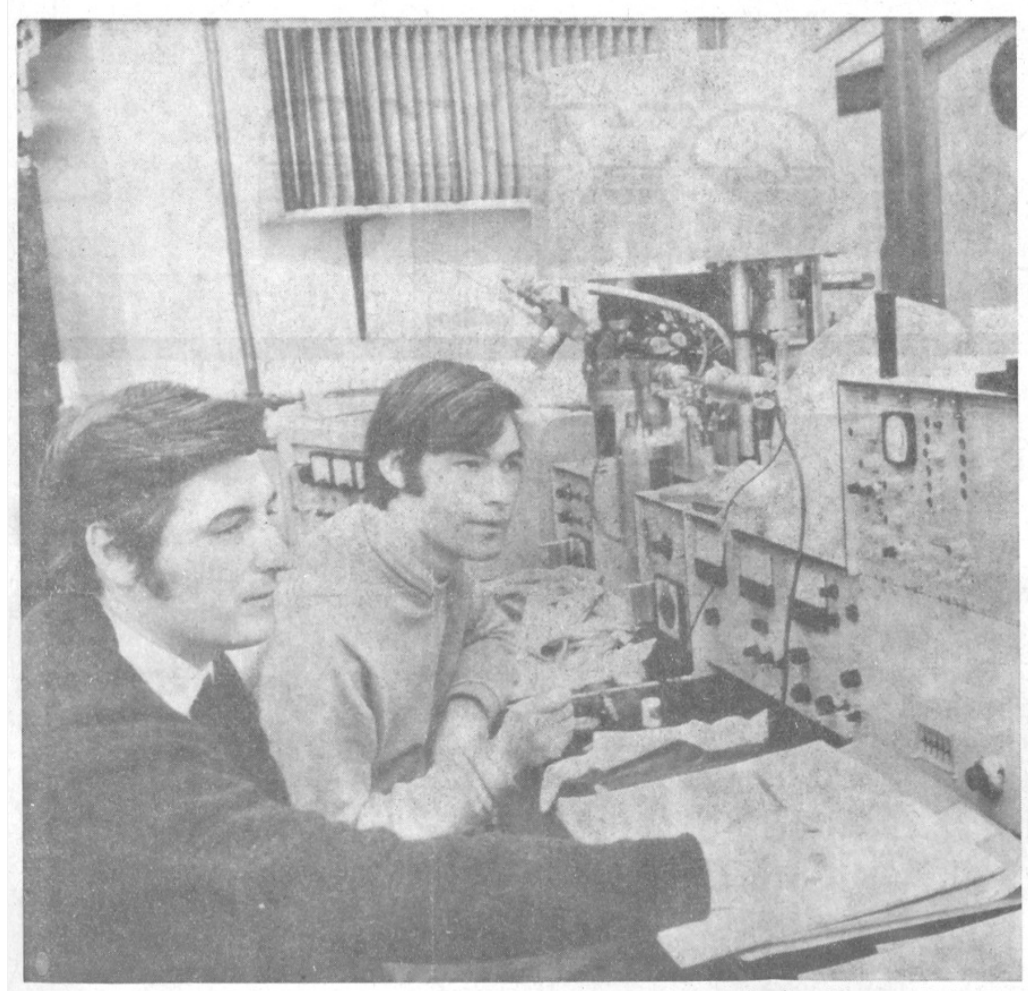

PR. DAVID CLARK and Dave Briggs, a research student, with the electron spectrometer in the science laboratory at Durham

\section{'Wonder' machine can trap burglars}

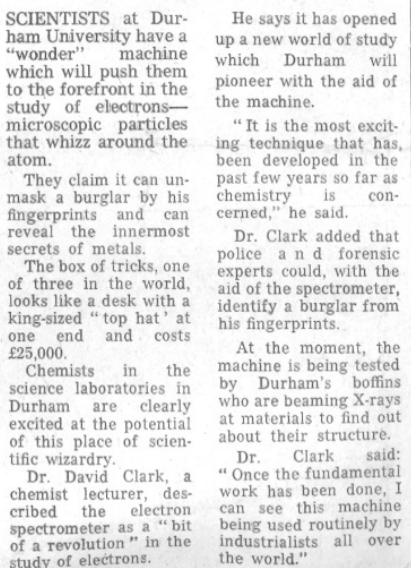

Figure 1

The first suggestion that XPS might have a future as a crime solving analysis method! The newly installed XPS spectrometer at the University of Durham, UK, autumn 1970. Reproduced by kind permission of the Evening Chronicle, Newcastle. 
(a)

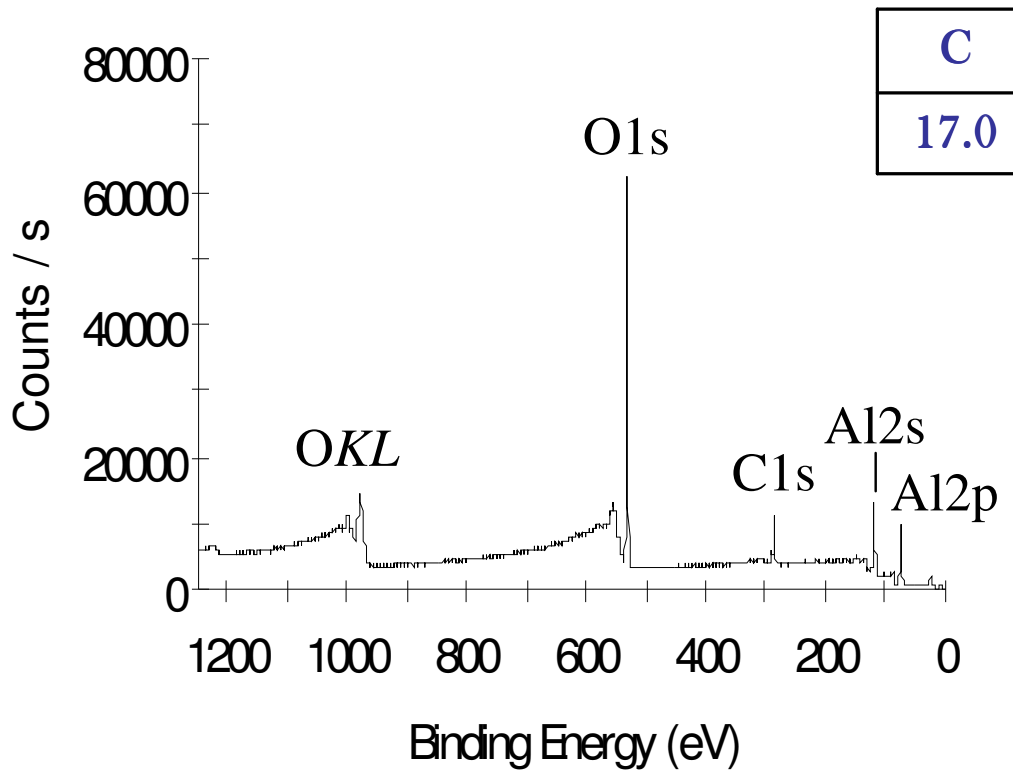

(b)

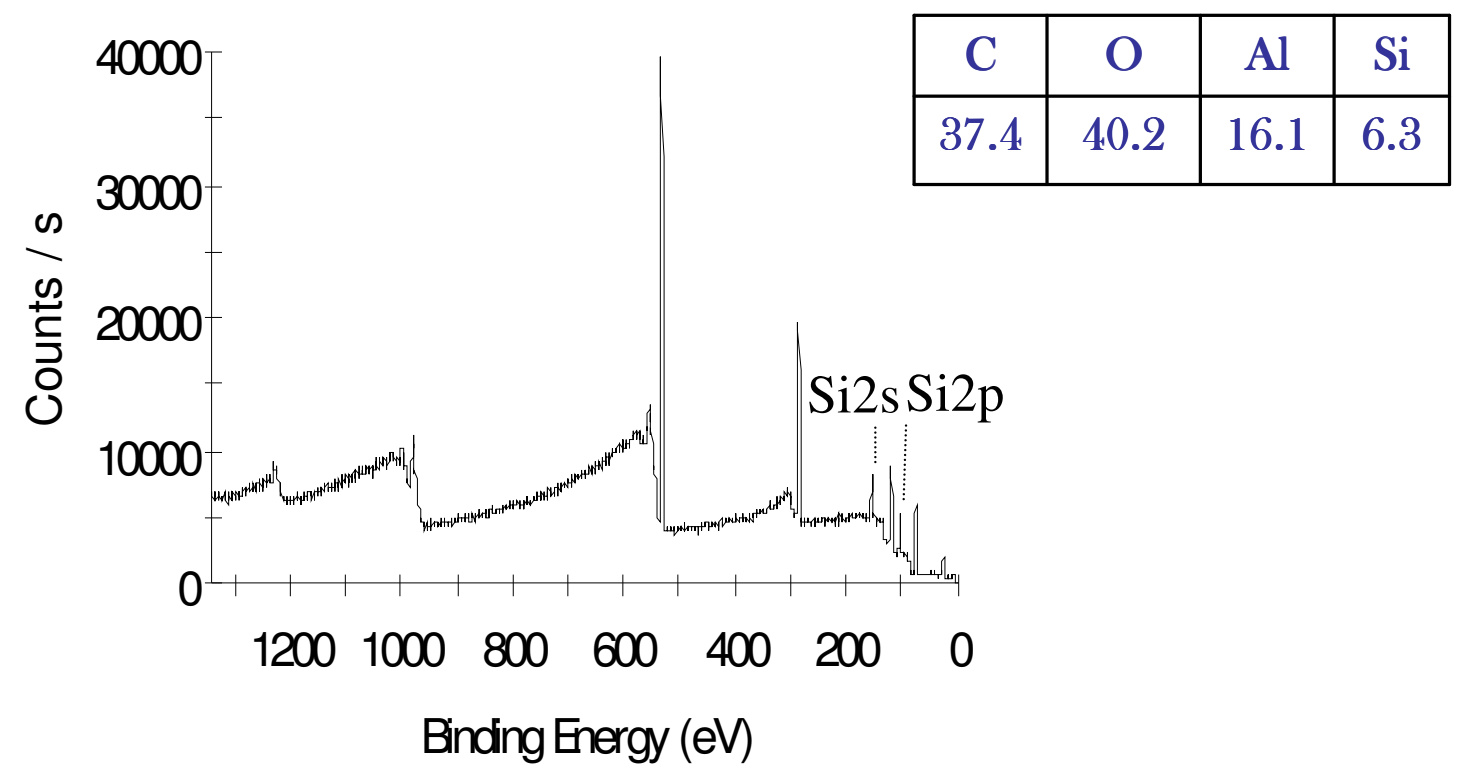

Figure 2

XPS survey spectra of clean aluminium foil and aluminium foil following handling. The Si2p and Si2s peaks in the latter spectra show the person touching the foil was a user of personal care products containing silicone oil. Quantitative surface analyses (in atomic $\%)$ are included in the boxes adjacent to the spectra. 


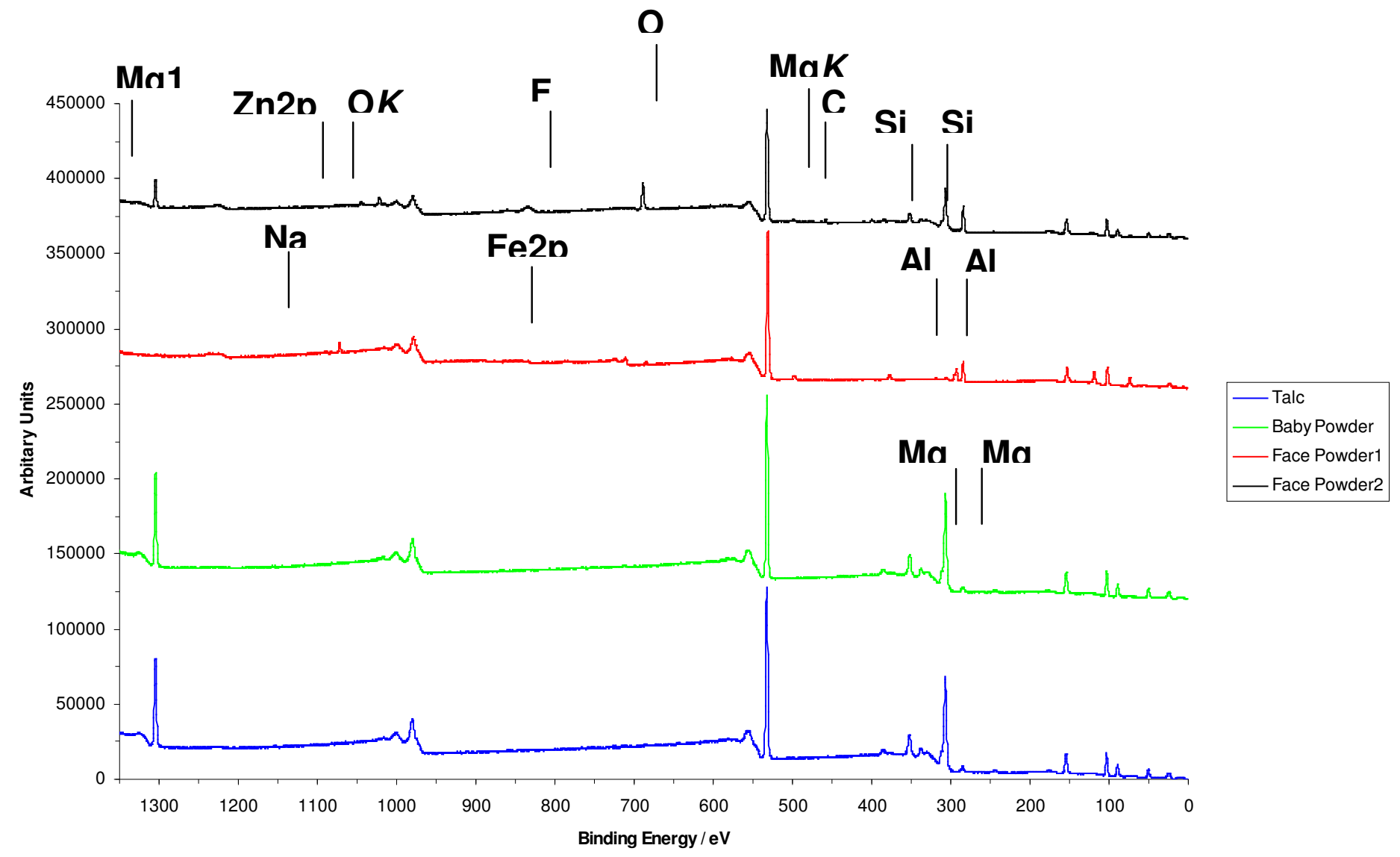

Figure 3

XPS survey spectra from talcum powder and a series of proprietary cosmetic powders.

\section{References}

M P Seah, S Spencer, R G White, Surf Interf Anal, 33, 960, (2002).

Proceedings of $14^{\text {th }}$ International Forensic Science Symposium, Interpol, Lyon, 19-22 October 2004, Ed N N Daéid,

M I Szynkowska, K Czerski, J Grams, T Paryjczak, A Parczewski, Imag Sci J, 55, 180, (2007).

S J Hinder, J F Watts submitted to Surf Interf Anal.

R Varga, S Zeman, M Kouba, J Hazad Mats A137, 1345, (2006)

D.R. Baer, A.S. Lea, J.D. Geller, J.S. Hammond, L. Kover, C.J. Powell, M.P. Seah, M. Suzuki, J.F.Watts, J.Wolstenholme, J Elec Spec, doi:10.1016/j.elspec.2009.03.021.

CERAM Surface and Materials Analysis, Application Note 2005:

http://www.csma.ltd.uk/latest_news/archive/forensic-science.htm

S J Hinder, J F Watts, University of Surrey, Unpublished data, July 2009.

H Uzawaa, K Ohgaa, Y Shinozakia, I Ohsawab, T Nagatsukac, Y Setob, Y Nishidad, Biosen, Bioelect, 24, 923, (2008).

10 B H Kaye, "Science and the Detective", VCH Press, Holland, 1995. 